\title{
Nasal alar deformity: a very rare complication of acoustic neuroma surgery after ipsilateral trigeminal and facial nerve paralysis*
}

Fotini leridou, Athanasia Printza, Zinovia Tsinaslanidou, Jannis Constantinidis

ENT, 1st ORL Department AHEPA HOSPITAL, Thessaloniki, Greece
Rhinology Online, Vol 1: 143 - 146, 2018 http://doi.org/10.4193/RHINOL/18.068

*Received for publication:

August 29, 2018

Accepted: October 4, 2018

Published: October 11, 2018

\begin{abstract}
Background: Nasal alar deformity (NAD) can affect both nasal breathing and the aesthetic appearance of the nose. NAD can be congenital or acquired. The acquired causes include nerve palsies, traumatic or iatrogenic. A rare condition that is associated with NAD is trigeminal trophic syndrome (TTS). TTS is an uncommon disorder of the trigeminal nerve characterized by a triad of facial ulcers, with anesthesia and paresthesia of the involved dermatomes. Nasal alar reconstruction is challenging for the surgeon and when it is performed, a delicate balance between aesthetic and functional outcome should be considered.
\end{abstract}

Case report: A 37 years old woman presented with nasal alar deformity caused by facial and trigeminal nerve paralysis after brain surgery. The woman was diagnosed with an acoustic neuroma and underwent complete tumor removal from neurosurgeons. After surgery, the patient suffered from facial and trigeminal nerve paralysis and 4-years later she presented with a progressive nasal alar deformity accompanied by breathing and aesthetic dysfunction. Reconstruction of the nasal ala was performed in one-stage surgery using spreader, alar batten and rim grafts. Seven years postoperatively, our patient had good nasal breathing and did not show any alar collapse.

Conclusions: In our case we believe that facial and trigeminal nerve palsies as a result of brain surgery, have both contributed to the deformity of the nasal ala. Reconstruction with the optimal technique and graft guaranteed long term results.

Key words: nasal alar deformity, acoustic neuroma, facial and trigeminal nerve palsies

\section{Introduction}

Nasal alar structural and functional integrity is very important for nasal breathing and facial aesthetic appearance. Nasal area is composed of bones, cartilages, muscles, nerves and ligaments. An important muscle of the nasal base is the levator labii alaque nasi muscle (LLANM) that is innervated by the zygomatic and superior buccal branch of facial nerve. The medial part of LLANM retracts laterally and superiorly to dilate nostrils. Another dilator of the nose is the dilator naris. In addition, the ophthalmic and maxillary branches of trigeminal nerve are responsible for nasal muscles sensitivity ${ }^{(1,2)}$. Weakness of the nasal valve results in nasal alar collapse. Common causes of nasal alar deformity include ageing, trauma, prolonged nasal obstruction, nasal surgery (rhinoplasty) and radiotherapy. Furthermore, nasal ala deformity can be attributed to neurogenic causes such as stroke or facial nerve paralysis ${ }^{(3)}$. Besides more common entities, a rare syndrome, trigeminal trophic syndrome (TTS) is associated with nasal ala deformity. TTS is an uncommon condition that results from trigeminal nerve injury. It is characterized by a triad of symptoms: paresthesia and anesthesia of the involved dermatome and facial ulceration in particular at the alar nasi region (4). Ulceration often occurs due to scratching and manipulation of the affected area ${ }^{(5)}$. The causes of TTS remain unclear. In two thirds of the cases, the etiology is organic (e.g. cerebrovascular accident), infection or cancer. Other causes are Wallenberg's syndrome (brain stem infarct) or iatrogenic i.e. post-removal of 


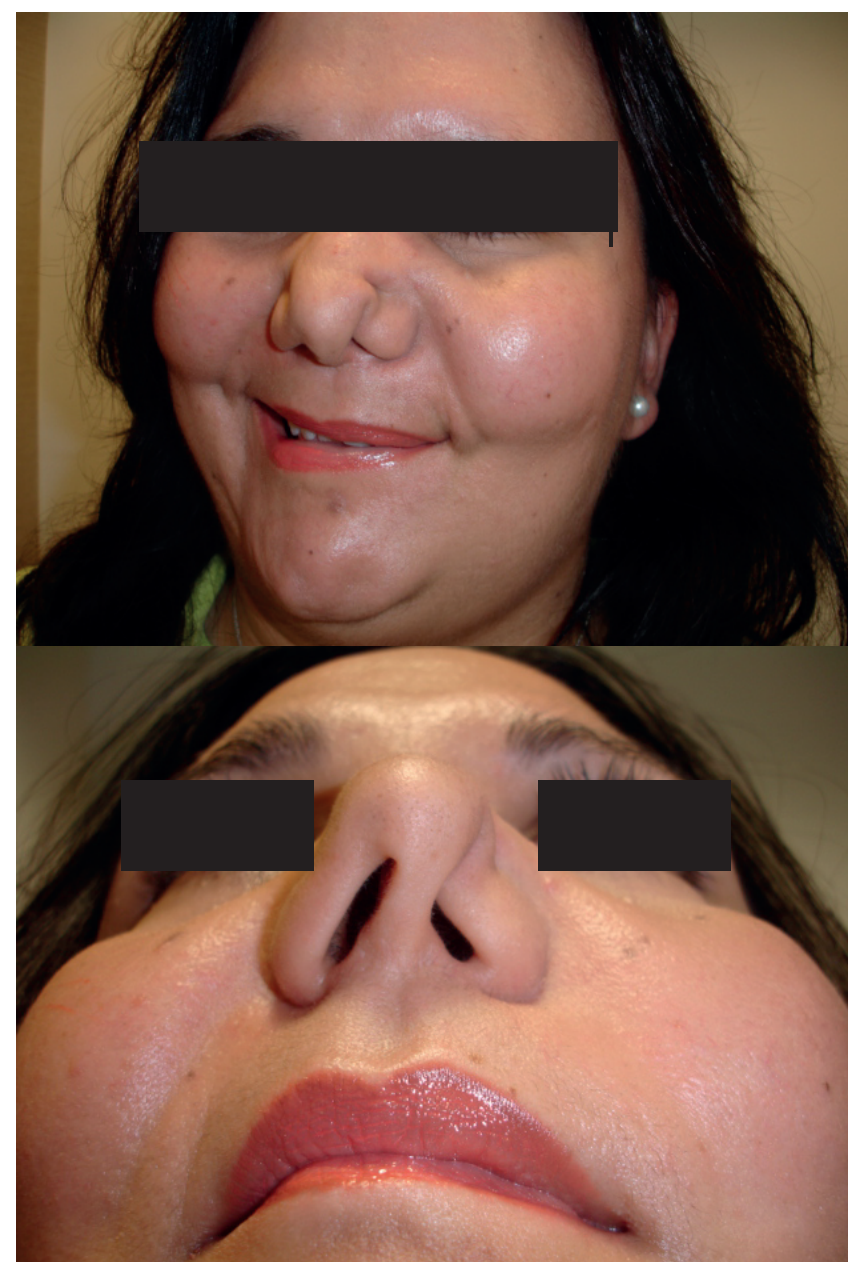

Figure 1. Preoperative three-quarter (a) and basal (b) view.

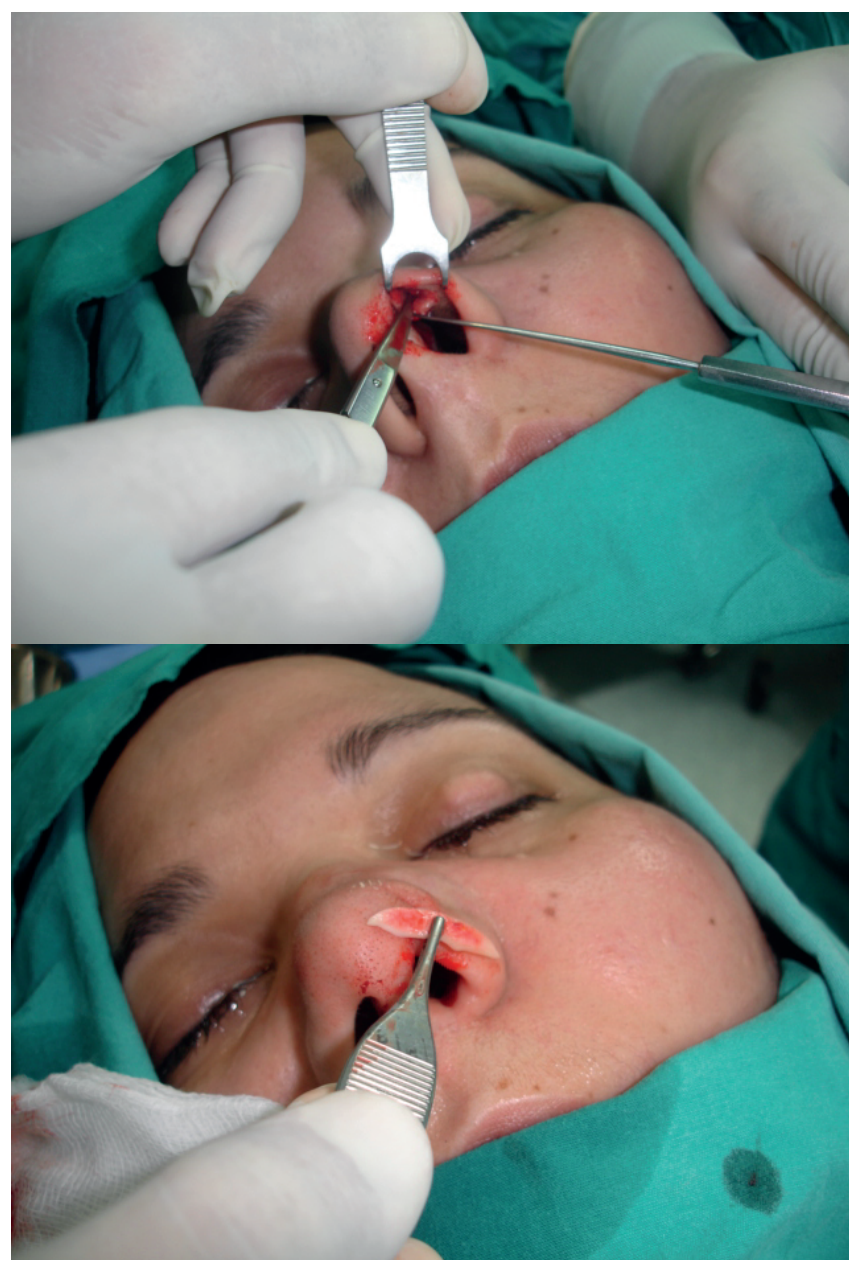

Figure 2. Intraoperative photos showing marginal incision (a) and placement of rim graft (b). acoustic neuroma ${ }^{(6,7)}$. As a result, nasal alar deformity (NAD), can affect patients' aesthetic appearance as well as nasal function; thus, patients may complain of nasal obstruction due to nasal valve narrowing ${ }^{(3)}$. We report a rare case of nasal alar deformity presenting as late post-operative complication of acoustic neuroma surgery. To our knowledge, the association of disorders of both trigeminal and facial nerve, after brain surgery with the clinical presentation of nasal ala deformity without ulcerations has not been previously reported.

\section{Case report}

A 37-years old woman presented with a nasal alar deformity on the left side without any history of ulceration of that particular area. Four years earlier she was diagnosed with an acoustic neuroma on the left cerebellopontine angle surgically treated via sub-occipital, retro-sigmoid approach. After surgery, the patient presented with left facial palsy and hemi- facial anesthesia. The patient underwent a cross facial nerve graft surgery to correct facial nerve palsy; however, the outcome wasn't satisfactory. Two years following the second surgery the woman presented with nasal shrinkage on the left side, with alar retraction and partial destruction of the left alar rim (Figure 1, $a-b)$. Although she described numbness on the left side of her face after the first surgery caused by trigeminal nerve palsy, the patient denied self- manipulation of her nose and there were no ulcerations at her nasal ala before the appearance of the deformity. She suffered from ipsilateral nasal breathing problems and was unsatisfied with her nasal appearance. Reconstruction of the left ala nasi and lateral nasal wall was conducted in one stage surgery. We did not use the classic external rhinoplasty approach with a columella skin incision in order to avoid skin incisions and reduce the risk of lesions and wounds that do not heal (associated to the trigeminal trophic syndrome). Instead, a spreader graft was placed in a sub-mucoperichondrial pocket via an inter-cartilaginous incision for the reconstruction of the internal nasal valve. Rim graft and long alar batten graft up to the piriform aperture were used via a rim incision in order to achieve a sufficiently stabilizing effect of the ala (Figure 2, a-b) The grafting material was harvested from conchal cartilage, because the patient previously underwent septoplasty. Seven 

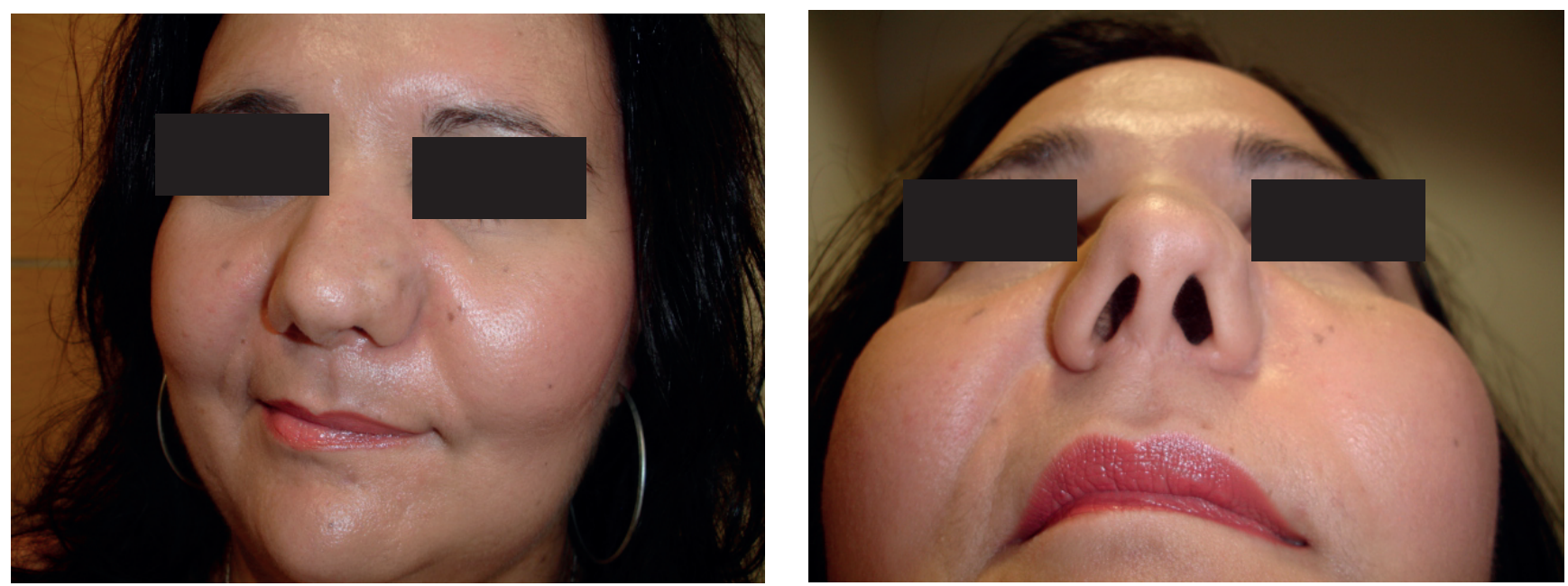

Figure 3. Postoperative three-quarter (a) and basal (b) view.

years postoperatively, our patient had good nasal breathing and did not show any alar collapse (Figure 3, a-b). The patient gave informed consent on this publication.

\section{Discussion}

The anatomy of nasal area is complex and aesthetic appearance and function depend on the optimal state of muscles, cartilages, nerves and skin. The most important dilator of nasal ala is LLANM $^{(2)}$. Alar retraction is one of the anatomical deformities that can affect both the aesthetic as well as the functional properties of the nose ${ }^{(8)}$.

There are several causes of nasal alar deformity: congenital or acquired ones. Acquired causes include traumatic, iatrogenic deformity or nerve palsies. Facial nerve palsy causes depression of alar base and loss of LLANM function. Alar collapse can also be attributed to the loss of dilator naris muscle function ${ }^{(9,10)}$. Another cause for nasal alar deformity is trigeminal nerve palsy. The etiology of trigeminal nerve injury varies and it involves trigeminal nerve ablation, cerebrovascular accidents, surgical complications, trauma and herpes zoster infection ${ }^{(5,6,11,12)}$. A rare condition that is associated with trigeminal nerve palsy is TTS.

TTS is developed after peripheral or central damage of the trigeminal nerve ${ }^{(13,14)}$. It was first described by Wallenberg in $1901^{(15)}$. The pathologic process that leads to the appearance of ulcers is not known yet. There is a neurotrophic theory stating that skin ulceration is due to the cessation of production of neurotrophic factors necessary for the maintenance of skin and other body parts well-being ${ }^{(4)}$. Another theory suggests that the ulceration is caused traumatically secondary to paresthesia by an unconscious self-manipulation. Self- induced trauma because of neurologic injuries and facial paresthesia causes ulceration that is difficult to heal (16).

However, a significant proportion of patients do not self-traumatize the anesthetic area of their face and therefore self-mani- pulation in not a pre-requisite for trigeminal trophic syndrome development ${ }^{(15)}$. The ulcers tend to appear in small areas and more often in the nasolabial crease and nasal ala area and less frequently they can spread onto the cheek and the upper lip. The tip of the nose is believed to be spared because its nerve supply is derived from the ethmoidal branch of the trigeminal nerve ${ }^{(14)}$. The period from the trigeminal nerve injury until the appearance of the ulcers varies from weeks to decades $(17,18)$. The treatment is challenging and should be individualized. It involves local wound care with antibiotics, hydrocolloid dressings and medications such as pimozide, amitryptiline, diazepam and carbamazepine, used to suppress paresthesia and prevent selfmanipulation of the ulcers ${ }^{(13,19)}$.

Defects of nasal ala are challenging to reconstruct due to its three-dimensional anatomy. The importance of reconstruction is to provide symmetry and maintenance of nasal function. Thus, a surgical approach using flaps and grafts has been attempted ${ }^{(20)}$. There are different flaps that can be used i.e. skin, composite and local ones. Alar batten grafts (ABG) (e.g. conchal) that we used in our case, provide structural support of the alar sidewall. $A B G$ are placed laterally to the crura as an overlay ${ }^{(21)}$.

Conchal cartilage grafts are good for nasal alar defects repair as they mimic the natural arch of ala and are elastic. Conchal grafts are autologous tissue grafts that have similar contour as the alar rim, maintaining nasal valve patency. Autologous cartilage grafts are recognized as the gold standard in nasal reconstruction. Examples include choncha, ribs or septal cartilage with good long-term results. Autologous grafts have better blood and nerve supply, resulting in better survival in comparison to allografts or grafts harvested from neuropathic facial area, which often are led to shrinkage of the graft and recurrence of skin ulceration. In our case to avoid ulcer formation or recurrence of alar deformity, the graft used for reconstruction was from conchal cartilage and an inter-cartilaginous approach and rim 
incision was used for reconstruction as the skin was atrophic due to nerve dysfunction ${ }^{(19-21)}$.

In the case we present there was no history of self-manipulation and no history of ulcerations. The clinical presentation was different from the typical triad of trigeminal trophic syndrome since there was no ulcer but only atrophy and shrinkage of the left nasal ala. The affected area presented muscle paralysis, cartilage shrinkage and skin atrophy. Skin was affected by trigeminal palsy as in trigeminal trophic syndrome although without ulcers. We believe in our case that the primary cause of the deformity of the nasal ala is the facial palsy which co-existed with trigeminal nerve palsy. This combination is a very rare complication of acoustic neuroma surgery. However, other conditions such as stroke or brain tumor can be associated with both nerve palsies. The facial nerve palsy caused the weakness of the facial muscles that lead to the atrophy of the nasal ala muscles which was later followed by atrophy of the cartilage and skin, leading to shrinkage of the nasal valve. We believe that the lesions in those two nerves have both contributed to the clinical presentation of our patient although the exact etiology path is not exactly clear yet. To our knowledge, the association of facial nerve palsy and trigeminal nerve palsy with nasal deformity without ulcerations has not been previously reported. The surgical repair resulted in full recovery and the patient, 7 years post operatively, had a great aesthetic and functional result. This is attributed to the absence of self-manipulation and the choice of optimal surgical technique (location of the skin incision, spreader graft reconstruction of the nasal valve and rim graft) and reliable and suitable grafting material (choncal cartilage).

\section{Conclusion}

We describe what we believe to be the first reported case of a deformity of nasal ala caused by facial and trigeminal nerve palsy, complications that have occurred after brain surgery. The detrimental effects of the two nerve palsies finally contributed to the shrinkage of the nasal ala: skin was affected in a way similar to trigeminal trophic syndrome, cartilage was thinned and alar muscles were paralyzed. Reconstruction of the nasal ala with optimal technique and graft resulted in good long-term results.

\section{Authorship contribution}

JC was involved in the Clinical work up of our case and commending on the manuscript; AP was involved in reading and commenting on the manuscript. ZT was involved in reading the manuscript IF was the author of the manuscript.

\section{Conflict of interest}

The authors have no conflicts of interest to declare.

\section{References}

1. Hur MS, Hu KS, Song WC, Abe S, Kim HJ New anatomical profile of nasal musculature: dilator naris vestibularis, dilator naris anterior and alar part of nasalis. Clin Anat. 2011; 24(2):162-7.

2. Taq S. Correcting the Alar base retraction in crooked nose by dissection of levator alaque nasi muscles. Ann Plast Surg 2016;72(4):383-387

3. Bruintjes T, Olphen A, Hillen B, Huizing E. A functional anatomic study of the relationship of the nasal cartilages and muscles to the nasal valve area. Laryngoscope. 1998; 108:1025-1032

4. Mishra SN, Nayak CS, Deshpande PJ, Pereira RR. Trigeminal trophic syndrome. A rare entity. Ind J Derm 2011; 77:729

5. 5. Willis M, Shockley WW, Mobley SR Treatment options in trigeminal trophic syndrome: a multi-institutional case series. Laryngoscope. 2011; 121 (4): 712- 716.

6. Sadeghi P, Papay FA, Vidimos AT. Trigemina trophic syndrome-report of four cases and review of the literature. Dermatol Surg. 2004; 30 (5): 807- 812

7. Koch M, Constantinidis J, Hornung J, Winter M. Das neurotrophische ulkus des N.trigeminus. HNO.2004;52;447-450

8. Guyuron B. Alar rim deformities. Plast Rec Surg. 2001;107(3):856-63.
9. Pessa JE, Crimmings CA. The role of facial muscle resection in reconstruc tion of the paralyzed face. Ann Plast Surg.1993;30(6):537-540.

10. Patel A, Knoll BI, Persing JA. A Congenital cleft of the alar rim. Plast Rec Surg.2009;123(2):67e-69e.

11. Westerlund $U$, Linderoth B, Mathiesen $T$. Trigeminal complications arising after surgery of cranial base meningiomas. Neurosurg Rev. 2012; 35 (2): 203- 209.

12. Levine JM. Historical perspective: the neurotrophic theory of skin ulceration. JAGS 1992; 40(12): 1281-1282.

13. Rashid RM, Khachemoune A. Trigeminal trophic syndrome. J Eur Acad Dermatol Venl. 2007; 21( 6): 725- 731.

14. Garza I. The trigeminal trophic syndrome an unusual cause of face pain, dysaesthesias, anesthesia and skin/soft tissue lesions. Cephalalgia. 2008; 28( 9): 980- 985.

15. Ferrara G, Argenziano G, Cicarelli G, Cusano F, Delfino M. Post-apopletic trigeminal trophic syndrome. J EUR Acad Derm Vener. 2000; 15(2):153-5.

16. McVeigh KA, Adams M, Harrad R, Ford R.Periocular manifestations of trigeminal trophic syndrome: A case series and literature review.Orbit. $2017 ; 16: 1-4$.

17. Weintraub E, Soltani K, Hekmatpanah J, Lorincz AL.Trigeminal trophic syndrome.
A case and review. J Am Acad Dermatol. 1982;6(1):52-7.

18. Fruhauf J, Schaider $\mathrm{H}$, Massone $\mathrm{C}$, Kerl $\mathrm{H}_{\text {, }}$ Mullegger RR.Carbamazepine as the only effective treatment in a 52-year-old man with trigeminal trophic syndrome. Mayo Clin Proc. 83(4):502-4.

19. Quatela VC, Jacono AA. Structural grafting in rhinoplasty. Fac Plast Sur.2002; 18:223-32.

20. Xavier R, Azeredo-Lopes S, Papoila A. Spreader grafts: functional or just aesthetical? Rhinology. 2015; 53(4):332-9.

21. Becker DG, Becker SS, Saad SS. Auricular cartilage in revision rhinoplasty. Fac Plast. Surg. 2003;19:41-51.

22. Munnoch DA, Morris AM. Trigeminal neuralgia, trophic ulceration and the plastic surgeon. J RCS End. 1998; 43:185-188.

Dr. Jannis Constantinidis

Department of Otorhinolaryngology

Head and Neck Surgery

Aristotle University of Thessaloniki

54006 Thessaloniki

Greece

E-mail: janconst@otenet.gr 